\title{
THE ANATOMY OF THE VOLAR PART OF THE CAPSULES OF THE FINGER JOINTS
}

\author{
Palle Gad, Århus, Denmark \\ From the Department of Anatomy, the Royal Dental College, Arhus, and \\ Department of Surgery, Arhus County Hospital
}

Rupture of the volar parts of the capsules of the finger joints has now been defined as a nosological entity within the group of subcutaneous lesions of the fingers. It has not been possible on the basis merely of studies of the literature to arrive at any satisfactory understanding of the correlation between anatomical conditions and clinical findings in such capsular ruptures.

The object of the present study has been to investigate in greater detail, macroscopically, the volar parts of the capsules of the finger joints and their relation to adjacent structures. The metacarpo-phalangeal joint of the thumb, recently described by Stener $(1962,1963)$ is not included in the present study.

\section{MATERIAL AND METHODS}

The investigation was carried out on the fingers of an adult hand that showed no signs of disease. The hand was fixed in a solution of 30 grammes of glycerine, 120 grammes of 96 per cent alcohol, 50 grammes of 36 per cent formalin, 10 grammes of phenol, 25 grammes of Epsom salt and 50 grammes of chloral hydrate in 1 litre of water.

Because conventional dissection did not give information of any value the fingers were dissected by the following technique: the specimen was placed in water for twenty-four hours in order to dilute the preservative and in particular the alcohol contained in it. Then the specimen was placed in a plastic bag together with a little water and frozen at -20 degrees Centigrade. With a power-driven bandsaw the frozen specimen was cut longitudinally into slices 2-3 millimetres thick (Fig. 1). No important loss of substance is involved in this procedure and the structures of the cut surface remain apparently uninjured (Fig. 2). After thawing the specimen is ready for examination.

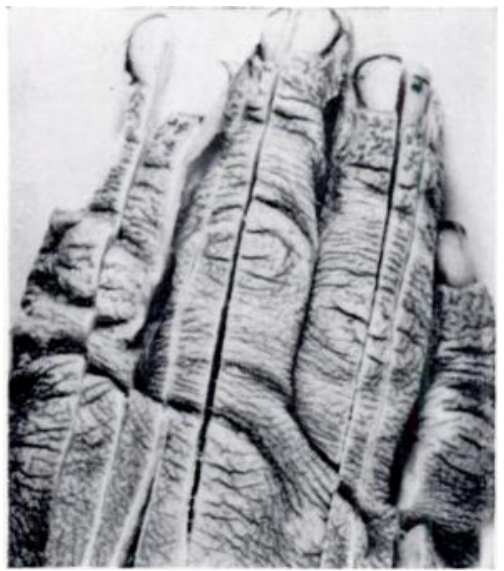

FIG. 1

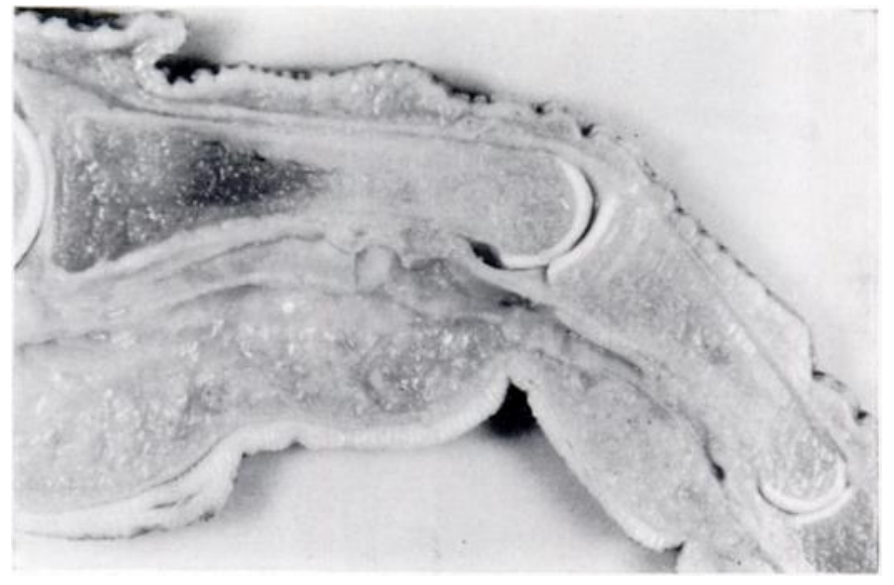

Fig. 2

Figure 1-The frozen specimen cut into slices by the band saw. Figure 2-Longitudinal, axial section through a finger showing the metacarpo-phalangeal joint, the proximal and distal interphalangeal joints and the tendon sheath without the flexor tendons. 


\section{RESULTS}

BONY ATTACHMENT OF ARTICULAR CAPSULES

Distal attachment-The following description applies to all joints examined. The fibrous capsule is attached to the narrow brim along the border of the articular cartilage at the phalangeal base (Fig. 3). At the site of attachment the volar part of this fibrous capsule is

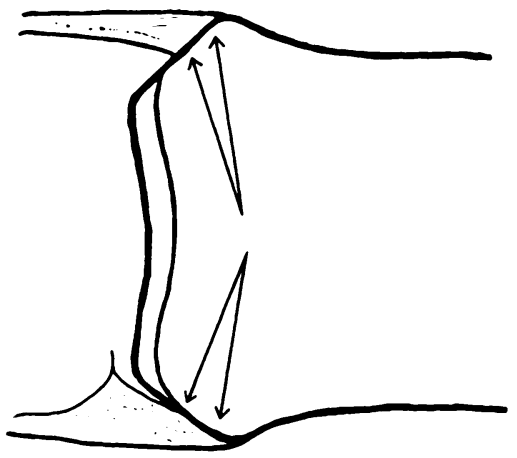

Fig. 3

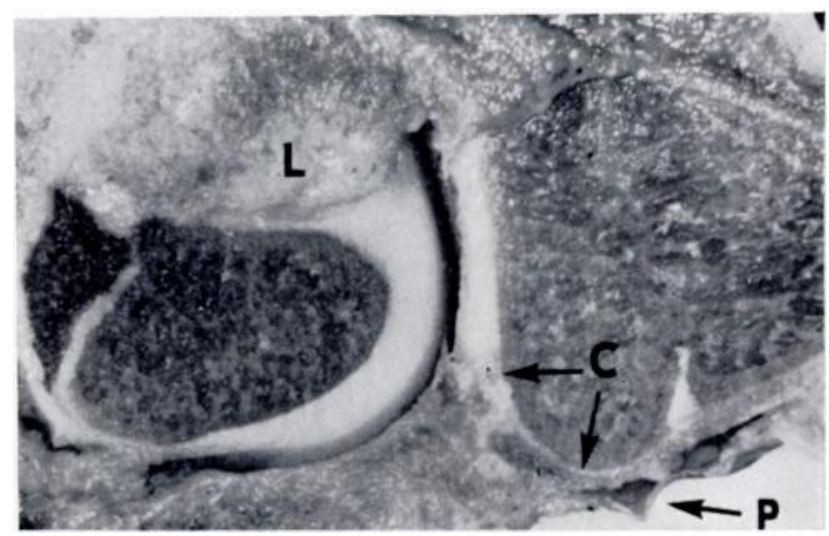

Fig. 4

Figure 3-Drawing of sagittal section through the distal part of the finger joint. Arrows indicate the attachment of the fibrous capsule. The external layers of the joint capsule continue on the surface of the phalanx. Figure 4Longitudinal section through lateral part of metacarpo-phalangeal joint. The distal, volar part of the joint capsule has a broad insertion into the bone (C). The periosteum (P) is exposed right to the site of insertion. The collateral ligament is cut close to its site of origin on the metacarpal head (L).

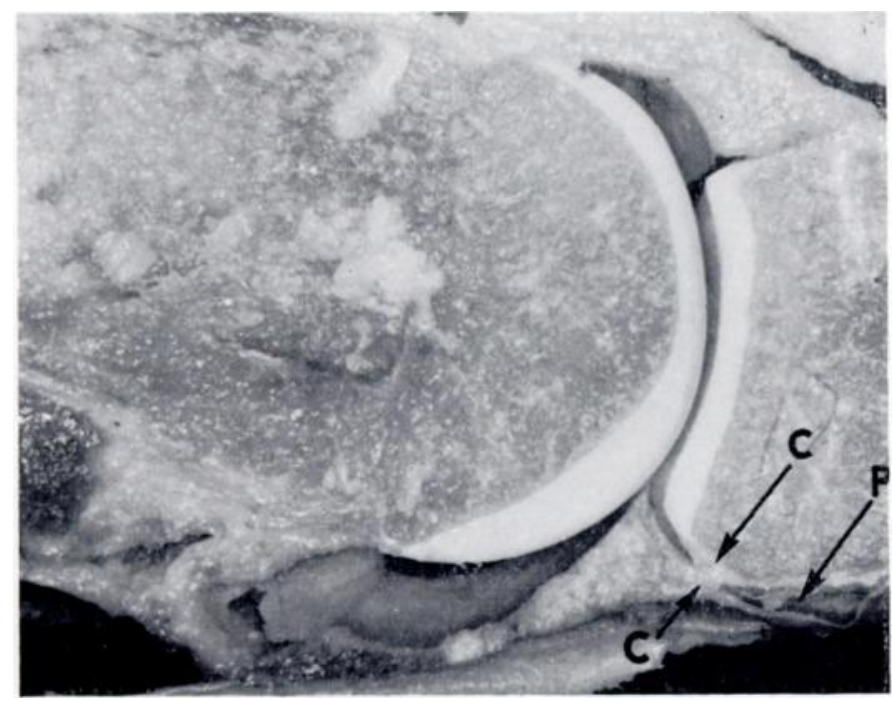

Fig. 5

Longitudinal, axial section through the metacarpo-phalangeal joint. The distal, volar part of the joint capsule inserts on a narrow rim $(C)$. The periosteum $(P)$ is exposed right to the site of insertion.

composed of two thick, lateral parts (Fig. 4) and one thin, axial part (Fig. 5). No synovial recess is found at the site of the distal attachment.

Proximal attachment-Two thick, lateral parts and one thin, axial part of the fibrous capsule are present also at the proximal attachment.

The metacarpo-phalangeal joints differ a little from the interphalangeal joints. At the metacarpo-phalangeal joint the proximal attachment of the capsule resembles the distal one 
except that a proximal synovial recess is present (Fig. 6). At the interphalangeal joints the fibrous joint capsule and the fibrous tendon sheath have fused (see below). The lateral parts of the capsule are attached to the bone immediately proximal to the head, whereas the axial part is indistinguishable from the fibrous tendon sheath. The axial, thin part of the proximal attachment is very lax; here the synovial membrane of the capsule forms a large recess (Fig. 7).

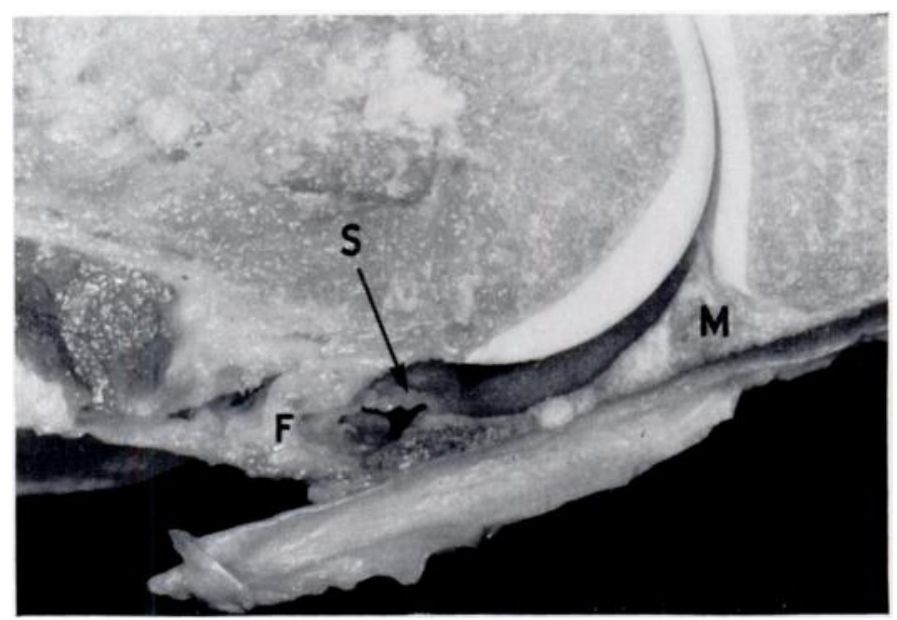

Fig. 6

Longitudinal. axial section through the metacarpo-phalangeal joint. At the site of the proximal insertion the joint capsule is separated into its two layers: the fibrous (F) and the synovial membrane (S). Part of the joint capsule $(\mathrm{M})$ resembles a meniscus. Note the tendon sheath and its proximal blind end.

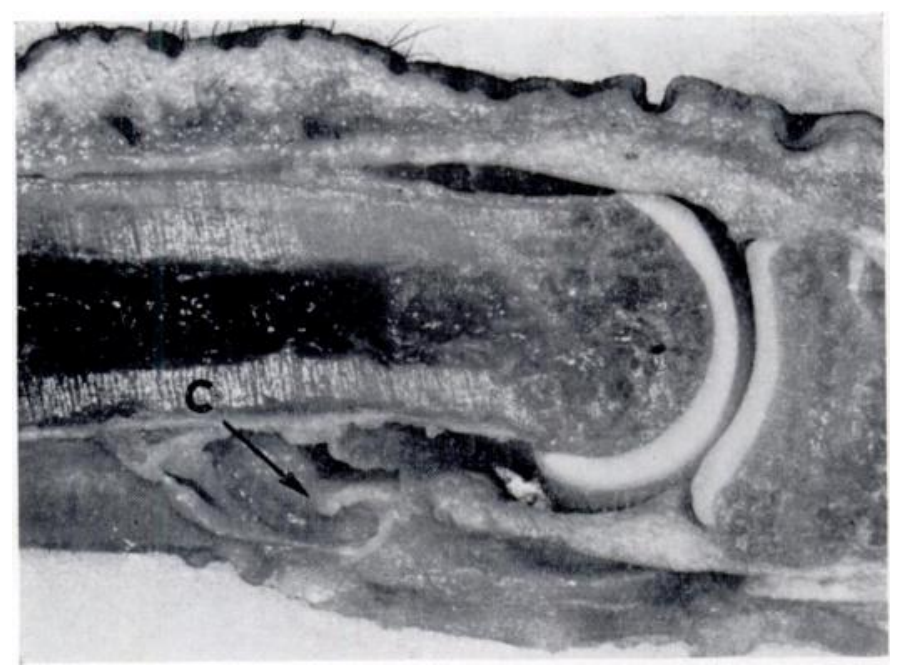

Fig. 7

Longitudinal, axial section through the proximal interphalangeal joint. The dorsal and volar synovial reflection folds are shown. The synovial tendon sheath is separated from the fibrous capsule which terminates proximally in a free border $(C)$.

In all of the interphalangeal joints the structures are easily separated by blunt dissection: the synovial membrane of the joint capsule is drawn dorsally (Fig. 7), the synovial membrane of the fibrous tendon sheath (vagina synovialis) is drawn forward. Thus it will be seen that there is no axial attachment proper of the isolated fibrous capsule. The fibrous components 
are seen to be arranged more and more laterally towards the proximal end. It is apparent from Figure 7 that the loose connective tissue during dissection may be compressed, thus leaving a proximally concave "free border."

\section{RELATION TO JOINT CAVITY}

The volar part of the joint capsule is seen on sagittal sections of the metacarpo-phalangeal joint as a triangular structure like a meniscus which in normal position occupies the space between the articular surfaces (Fig. 6).

The photograph illustrating a "dislocated" first phalanx shows that the "meniscus" extends more than half way up on the inside of the joint capsule (Fig. 8). This feature, although less distinct, is found also in the interphalangeal joints.

\section{RELATION TO THE FIBROUS TENDON SHEATH}

The digital bones represent the major part of the deep boundary of the tendon sheath; at the site of the joints this boundary is formed by the volar part of the joint capsule. Figure 3 shows how the

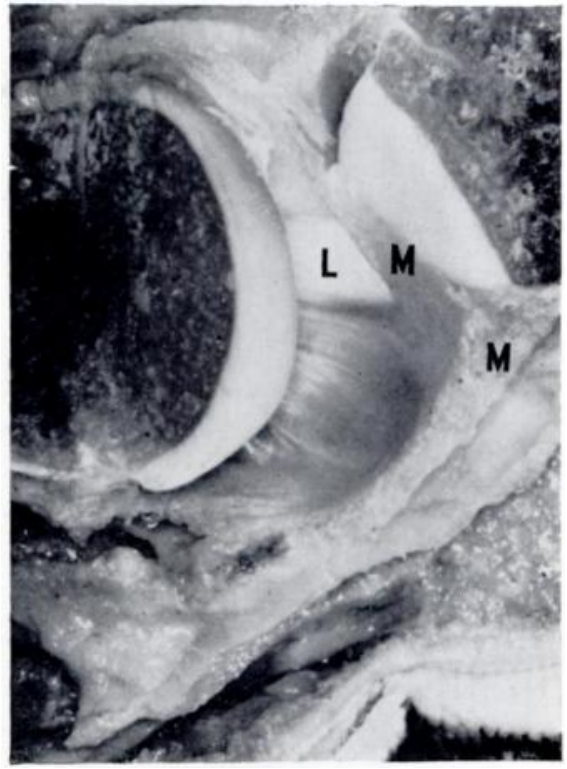

Fig. 8

Longitudinal, axial section through the metacarpo-phalangeal joint, in "dislocated" position, showing the inside of the capsule and the collateral ligament $(\mathrm{L})$. The "meniscus" $(\mathrm{M}, \mathrm{M})$ can be followed far up on the inside of the capsule. Note the tendon sheath and its proximal blind end in front of the capsule. outline of the bone continues uninterrupted in the outline of the joint capsule at the distal attachment.

Proximal to the joint the structure is more complicated. The tendon sheath is firmly attached to the periosteum along the lateral borders of the volar surfaces on the phalanges.

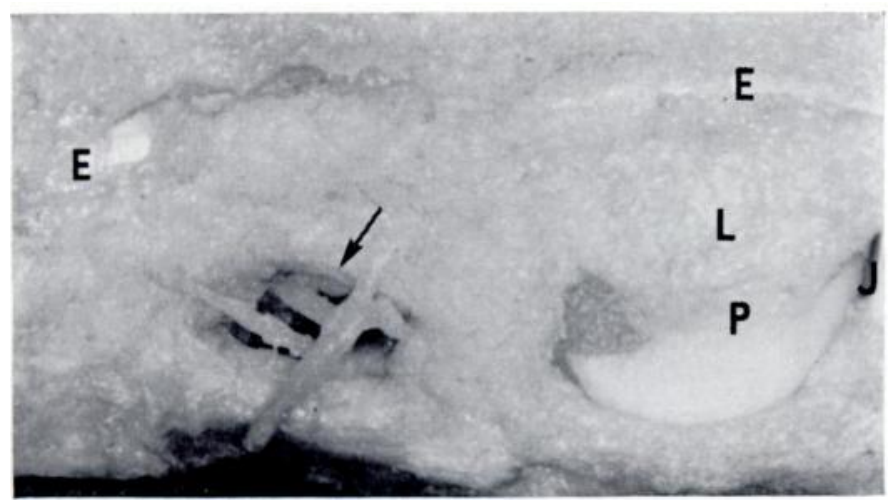

FIG. 9

Longitudinal section through the most lateral part of the proximal interphalangeal joint $(J)$; the intact head of the middle phalanx $(P)$ is seen. The margin of the extensor aponeurosis (E-E) and the cut collateral ligament $(\mathrm{L})$ are also seen. The arrow points to the opening between bone and tendon sheath in which an artery and vein are visible.

Just proximal to the interphalangeal joints, however, an opening measuring a few millimetres separates the lateral margins of the bone from the tendon sheath. Dorsally this opening is bordered by the bone; distally by the attachment of the joint capsule; in front by the fibrous 
tendon sheath; and proximally by the bony attachment of the tendon sheath (Fig. 9). Traces left by these attachments and their interval are well marked in bones from which the soft tissue has been removed (Fig. 10).

Through the opening it is possible to reach the cleavage between the volar surface of the bone and the deep part of the synovial tendon sheath. Laterally access to the subcutaneous tissue is easily obtained. In Figure 9 an artery and a vein are shown supplying the bone as well as other structures.

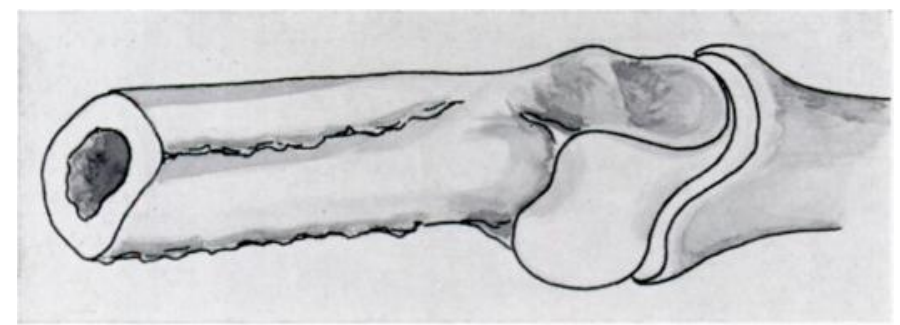

FIG. 10

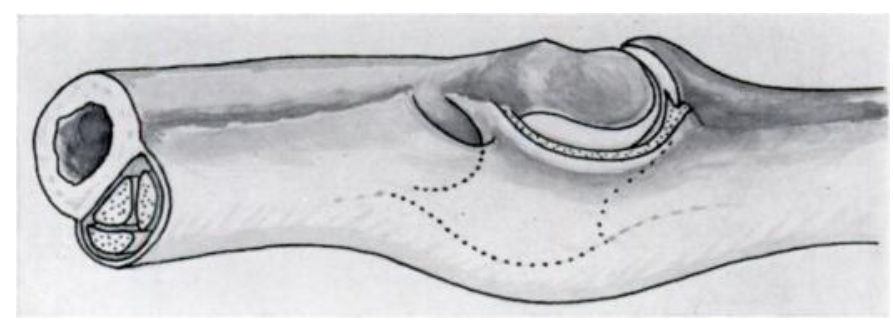

Fig. 11

Figure 10-Drawing of the bone with the remnants of the insertions of the tendon sheath and fibrous capsule. Figure 11-Drawing of the bone with the tendon sheath and joint capsule in place. The joint is cut open and the lateral capsule is "removed." (Drawings by Dr Bent Barfod.)

\section{RELATION TO TENDON INSERTIONS}

The flexor profundus tendon is attached mainly to the volar surface of the distal phalanx. A few fibres are inserted into the joint capsule, extending as far proximal as to the joint plane; the synovial sheath extends to this site on the dorsal aspect of the tendon while on the volar aspect reaches it farther distally.

The flexor superficialis tendon is attached to the volar surface of the middle phalanx; fibres extending to the proximal interphalangeal joint capsule have not been demonstrated.

\section{DISCUSSION}

The technique used for preparation offers many advantages. On serial sections of one finger all structures become easily accessible for investigation and may be followed throughout the finger. Thus the material is most thoroughly exploited and the investigation may be carried out on few fingers. Dissection entails merely the blunt separation of the structures because the thin specimens make it unnecessary to remove any tissue; moreover it is possible to reconsider structures already examined. The thin specimens are flexible and hence joints may be dislocated to permit direct inspection of the walls of the joint cavity.

The drawings presented by Moberg and Stener (1953) illustrate the recent conception of the gross anatomy of the volar part of the finger joints. The present investigation shows, however, that variations are even more numerous. This applies in particular to the attachment of the joint capsules to the bones as well as the relation between joint capsules and tendon sheaths. 
Moberg and Stener tried to provide an explanation of the three signs by which the diagnosis of rupture of the volar capsule of the finger joint may be established. It is generally accepted that extension and flexion of the finger give pain at the site of the lesion, whereas there is no pain when the finger is in semi-flexion. Distinct tenderness and probably bruising too are found. According to Moberg and Stener this triad may be explained by a tightening of " the volar ligament " on extension and flexion, while the absence of pain is explained by a relaxation of the "ligament" in semi-flexion.

The results obtained in the present study, however, support the conception that the proximal, flexible part of the "volar ligament" described by Moberg and Stener is less important than the other relations of the joint capsule to bone and tendon sheath. The structures of importance in producing symptoms are the solid, supporting and fixing lateral strands in the joint capsule rather than the relatively weak and yielding axial portions. The two drawings present the details as they have been found by the author (Figs. 10 and 11).

These observations raise certain questions about the anatomical point of action of hyperextension injuries and the exact aspect and localisation of the lesions. It is planned to give answers to these questions in later studies.

During the present investigation the author has had certain doubts whether it is justified to use the term "volar ligament" because the term "ligament" should be applied only to independent structures composed of solid connective tissue in which the arrangement of the fibres is orientated in one direction. The volar parts of the capsules of the finger joints do not fulfil this requirement. This problem too needs further investigation.

The opening between finger bones and tendon sheaths serves, as already mentioned, as passage for vessels to bone and joint capsule. This is also the site at which the otherwise firmly fixed and relatively rigid tendon sheath is flexible.

\section{SUMMARY}

1. A method of finger dissection is described which provides a new approach to the anatomical study of structures in close relation to joints.

2. The volar part of the capsule of the finger joints is described, the attachment to the bones being particularly emphasised together with its form which is like that of a meniscus.

3. A gap between bone and tendon sheath is described.

4. Theoretical and clinical aspects of the local anatomy are discussed.

\section{REFERENCES}

Moberg, E., and Stener, B. (1953): Injuries to the Ligaments of the Thumb and Fingers. Diagnosis, Treatment and Prognosis. Acta Chirurgica Scandinavica, 106, 166.

Stener, B. (1962): Displacement of the Ruptured Ulnar Collateral Ligament of the Metacarpo-phalangeal Joint of the Thumb. Journal of Bone and Joint Surgery, 44-B, 869.

Stener, B. (1963): Hyperextension Injuries to the Metacarpophalangeal Joint of the Thumb-Rupture of Ligaments, Fracture of Sesamoid Bones, Rupture of Flexor Pollicis Brevis. An Anatomical and Clinical Study. Acta Chirurgica Scandinavica, 125, 275. 